\title{
Obstructive sleep apnea predicts risk of metabolic syndrome independently of obesity: a meta-analysis
}

Yingjun Qian, Huajun Xu, Yuyu Wang, Hongliang Yi, Jian Guan, Shankai Yin

Department of Otolaryngology - Head and Neck Surgery, Shanghai Jiao Tong University Affiliated Sixth People's Hospital, Shanghai, China

Submitted: 23 January 2015

Accepted: 6 April 2015

Arch Med Sci 2016; 12, 5: 1077-1087

DOI: 10.5114/aoms.2016.61914

Copyright $\odot 2016$ Termedia \& Banach

\section{Abstract}

Introduction: Obstructive sleep apnea (OSA) has been suggested to be associated with a high risk of metabolic syndrome (MS). However, results on whether the association between OSA and risk of MS is independent of obesity, and the effect of nocturnal intermittent hypoxia (IH) on MS, are conflicting. Our purpose was to estimate the magnitude of the independent association between OSA and risk of MS and further explore whether nocturnal IH in OSA plays a role in MS risk.

Material and methods: The PubMed and EMBASE databases were systematically searched (until January 21, 2015) for available observational evidence. Unadjusted and body mass index (BMI)-adjusted pooled odds ratios (ORs) for MS in OSA or higher nocturnal IH were calculated using fixed or random models. Tests of homogeneity, publication bias, and robustness of the results were performed.

Results: A total of 13 independent studies (involving 857 participants in 3 case-control studies and 7077 participants in 10 cross-sectional studies) were included. The OSA was significantly associated with an increased risk of MS in a meta-analysis of 10 studies (pooled OR $=1.72,95 \% \mathrm{Cl}: 1.31-$ $2.26, p<0.001)$, with a BMI-adjusted pooled OR of 1.97 (95\% Cl: 1.34-2.88, $p<0.001)$. Pooled results from 3 studies on the oxygen desaturation index $(\mathrm{ODI})$ and $\mathrm{MS}$ risk $(\mathrm{OR}=1.96,95 \% \mathrm{Cl}: 1.73-2.22, p<0.001)$ and 3 studies on the cumulative percentage of sleep time with $\mathrm{SpO}_{2}$ below $90 \%$ (CT90) and $\mathrm{MS}$ risk $(\mathrm{OR}=1.05,95 \% \mathrm{Cl}: 1.02-1.07, p<0.001)$ were also significant. Conclusions: Our findings demonstrated a significant association between OSA and increased MS risk independent of BMI, and further indicated a role of nocturnal IH in this association.

Key words: intermittent hypoxia, sleep-disordered breathing, syndrome X, systematic review.

\section{Introduction}

Metabolic syndrome (MS) is a constellation of metabolic abnormalities including obesity, hypertension, hypertriglyceridemia, low high-density lipoprotein cholesterol, and hyperglycemia. According to the National Cholesterol Education Program Adult Treatment Panel III (NCEP ATPIII), coexistence of three of these components characterizes MS [1]. Metabolic syndrome is known to independently increase the risk of cardiovascular diseases (CVD) and all-cause mortality, even more than single MS components [2]. Because traditional lifestyle components such as diet and physical activity [3] might not fully account for the development of

\author{
Corresponding author: \\ Jian Guan MD, PhD \\ Department of \\ Otolaryngology \\ Head and Neck Surgery \\ Shanghai Jiao \\ Tong University \\ Affiliated Sixth \\ People's Hospital \\ 600 Yishan Road \\ 200233 Shanghai, China \\ Phone/fax: +862164834143 \\ E-mail: guanjian06@sohu.com
}


MS, other factors associated with MS need to be clarified. It is noteworthy that sleep disorders including impaired sleep quality, sleep deprivation, and sleep apnea have been reported to play key roles in the development of MS and its components [4-6].

Obstructive sleep apnea (OSA) is a respiratory condition characterized by repetitive hypopneas and apneas during sleep due to upper airway obstruction [7], affecting about $4 \%$ of males and $2 \%$ of females in the middle-aged population [8]. Obstructive sleep apnea has been associated with numerous cardiovascular risk factors [9], including MS. The association between OSA and risk of MS was previously thought to be related to obesity, which is their common risk factor. However, mounting evidence from epidemiology and experimental studies indicates that OSA itself is associated with increased risk of MS.

Experimental studies have demonstrated that OSA is not only associated with repeated cessation of breathing while sleeping, but is also accompanied by nocturnal intermittent hypoxia (IH) and micro arousal-related deregulation of the sympathetic nervous system, oxidative stress, and chronic inflammation [10, 11]. Interestingly, nocturnal IH has been found to be involved in the pathogenesis of impaired glucose hemostasis, hyperlipidemia, hypertension, and obesity in animal and cell models [12-18]. Epidemiology and intervention studies have also shown that OSA is an independent risk factor for MS components such as hypertension [19, 20], dyslipidemia [21, 22], and impaired glucose tolerance $[23,24]$.

Recently, observational human studies have reported an association between OSA and overall risk of MS. To date, 11 papers have been published $[6,25-34]$. Some of these studies also investigated the association between nocturnal $\mathrm{IH}$ and MS risk [31-34]. However, their results, especially after controlling for confounding factors, are inconsistent. Some of the papers reported positive independent associations between OSA or nocturnal IH and MS [25, 26, 28, 30, 32-34], while other papers reported non-significant associations [6, 27, 29, 31]. Although discrepancies and insufficient statistical power across these individual studies exist, no studies have quantitatively reviewed the association between OSA and MS risk, or the association between nocturnal $\mathrm{IH}$ and MS risk. Additionally, whether the associations between OSA, nocturnal $\mathrm{IH}$, and MS risk are due to the confounding effects of BMI has not been determined definitively.

Given that MS is a serious collection of risk factors for CVD morbidity and mortality, as well as the possible role of OSA and its related nocturnal $\mathrm{IH}$ in the risk of MS, we conducted a meta-anal- ysis to comprehensively clarify the independent association between OSA, nocturnal $\mathrm{IH}$, and risk of metabolic syndrome. We also evaluated the influences of BMI and other confounding factors.

\section{Material and methods}

The present meta-analysis was conducted according to the Preferred Reporting Items for Systemic Reviews and Meta-Analyses (PRISMA) statement [35].

\section{Search strategy}

Literature databases including PubMed and Embase were systemically searched (date range until January 21,2015$)$. To identify all possible studies, we used the following search terms: "sleep disordered breathing OR obstructive sleep apnea OR nocturnal intermittent hypoxia" AND "metabolic syndrome OR insulin resistance syndrome OR syndrome X." Abbreviated forms of these terms were also used. The reference lists of retrieved papers and relevant reviews were further hand-searched. The literature search was limited to human-based studies that had English editions.

\section{Eligibility criteria}

The studies included in the meta-analysis met all of the following inclusion criteria: (1) evaluated the association between OSA or nocturnal IH and risk of MS; (2) MS was considered as an outcome and OSA or nocturnal $\mathrm{IH}$ was considered as an influencing factor in the statistical analyses; (3) provided sufficient data for calculation of an odds ratio with a 95\% confidence interval; (4) recruited only samples aged $\geq 18$ years; (5) diagnosed OSA by polysomnography (PSG) and categorized samples with or without OSA according to the apnea hypopnea index $(\mathrm{AHI})$ or respiratory disturbance index (RDI), or categorized nocturnal IH by objective variables; (6) diagnosed MS by a certain published guideline; (7) had an English edition. If multiple papers reported data from the same set of samples, we retained only the one with the most detailed information to avoid duplicate results.

\section{Study selection and data extraction}

Preliminary screening for identifying potentially relevant studies against inclusion criteria was conducted by reading titles and abstracts. Final eligibility was determined by reviewing the full manuscripts of possibly relevant studies. We pre-built a data collection template for recording key items from the admitted studies. This included first author; publication year; country; study design; samples' characteristics; methods used to assess OSA, nocturnal $\mathrm{IH}$, and MS; and main results. We also 
extracted information for a quality evaluation of studies according to the Newcastle Ottawa Scale (NOS) [36]. Finally, for papers in which subjects were divided into separate groups by gender, each individual set of data was included in the meta-analysis [27, 34]; for papers that reported associations separately by different degrees of OSA severity or nocturnal $\mathrm{IH}$, the Hamling method [37] was applied to combine the odds ratios $[29,34]$. Two authors (Qian and Xu) independently assessed the articles for compliance with the inclusion criteria and extracted data. Discrepancies were resolved through discussion with a third author (Guan).

\section{Statistical analysis}

We chose the ORs adjusted for confounding factors, e.g., age, gender, and body mass index (BMI), for multivariable analyses with MS as the outcome and OSA or nocturnal IH as the independent factor. If no adjusted ORs were available, raw ORs were used. The associations between OSA or nocturnal $\mathrm{IH}$ and risk of MS were estimated by calculating pooled $\mathrm{OR}$ and $95 \% \mathrm{Cl}$ for MS, with significance determined by the $Z$ test $(p<0.05$ was considered statistically significant). To further evaluate the influence of controlling for $\mathrm{BMI}$ in the association between OSA or nocturnal $\mathrm{IH}$ and risk of MS, the BMI-adjusted pooled ORs for MS were also evaluated. Between-study heterogeneity was estimated by calculating the $1^{2}$ statistic and Q test $[38,39]$. A random- or fixed-effect model was applied to calculate pooled OR in the presence ( $Q$ test, $p<$ 0.10 ) or absence ( $Q$ test, $p \geq 0.10$ ) of heterogeneity. Meta regression and stratified analyses were conducted to examine the sources of heterogeneity.

The quality of each individual study was assessed using NOS [36]. Studies were graded on an ordinal star scoring scale, with higher scores representing studies of better quality. A maximum of nine stars could be given for each study. The quality of each study was graded as either level 1 (0 to 5 ) or level 2 (6 to 9). Sensitivity analysis was carried out omitting one study at a time to assess the stability of the results. Publication bias was assessed using Egger's test [40]. If publication bias was identified, the trim and fill method was applied to correct it by imputing data from presumed missing studies and reassessing the stability of the pooled ORs [41]. All analyses were conducted using the STATA software program ver. 12.0 (Stata Corp, College Station, TX, USA).

\section{Results}

\section{Study selection}

Figure 1 presents a flow chart of the literature searches and detailed inclusion/exclusion reasons.
A total of 2,314 articles were initially identified from the electronic databases. Preliminary screening excluded 2,299 articles, leaving 15 articles as potentially relevant. After full text review, 4 articles were further excluded because they were duplicates or evaluated OSA or nocturnal IH using methods that were not objective (e.g., questionnaires). Eleven articles were eligible for the final statistical analyses [6, 25-34]. All provided suitable data for pooled estimation of ORs. Because 2 of the 11 eligible papers reported ORs separately by gender [27, 34], these data were included in the meta-analysis as individual studies, which brought the final number of included studies to 13 .

\section{Study characteristics}

The 13 studies were all observational surveys, involving 3101 participants (2471 participants in 8 cross-sectional studies and 630 in 2 case-control studies) on the association between OSA and risk of MS [6, 25-32] and 5392 participants (5165 in 4 cross-sectional studies and 227 in 1 case-control study) on the association between nocturnal IH and risk of MS [31-34]. The publication years ranged from 2004 to 2012. A total of 3 studies were from the United States, 4 from Europe, and 6 from Asia. Overall, 8 studies used standard PSG to detect OSA, and 2 used portable PSG that lacked an electroencephalogram (EEG);

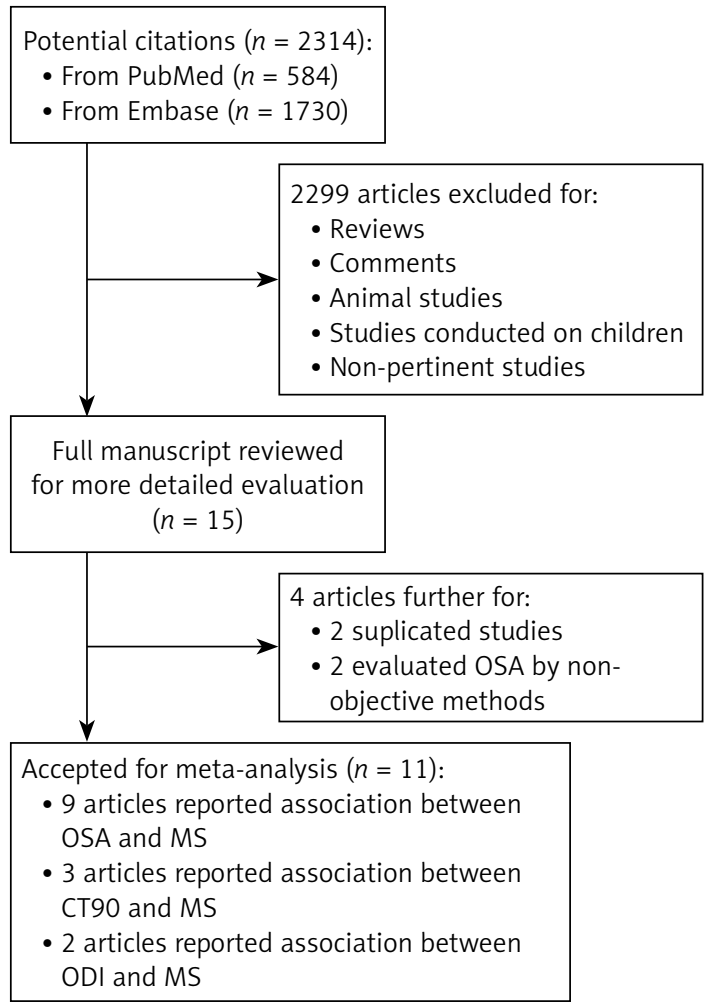

Figure 1. Flow chart of the systemic literature review: a total of 16 studies (in 11 articles) were included to perform the meta-analyses 


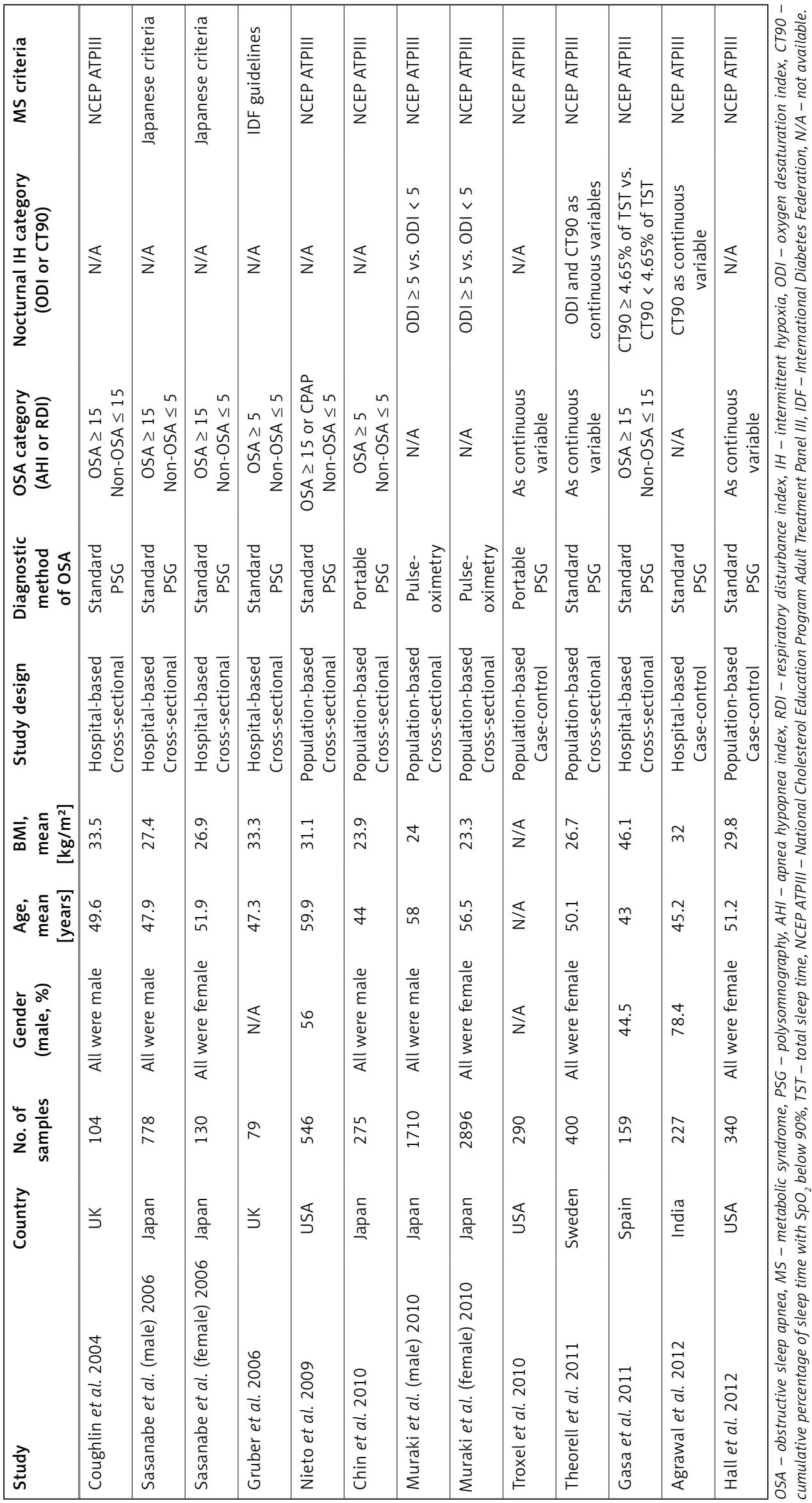


Table II. Lists of adjusted confounding factors in calculation of odds ratios

\begin{tabular}{|c|c|}
\hline Study & Adjusted confounding factors \\
\hline Coughlin et al. 2004 & Age, gender, BMI, alcohol consumption, smoking habits \\
\hline Sasanabe et al. (male) 2006 & Age, gender, BMI \\
\hline Sasanabe et al. (female) 2006 & Age, gender, BMI \\
\hline Gruber et al. 2006 & Age, BMI, smoking habits \\
\hline Nieto et al. 2009 & Age, gender, BMI \\
\hline Chin et al. 2010 & Age, BMI \\
\hline Muraki et al. (male) 2010 & $\begin{array}{c}\text { Age, smoking status, current alcohol intake, time since last meal, menopausal } \\
\text { status }\end{array}$ \\
\hline Muraki et al. (female) 2010 & $\begin{array}{c}\text { Age, smoking status, current alcohol intake, time since last meal, menopausal } \\
\text { status }\end{array}$ \\
\hline Troxel et al. 2010 & $\begin{array}{c}\text { Age, gender, race, marital status, study randomization, smoking status, alcohol } \\
\text { consumption, sedentary lifestyle, presence of clinically significant depressive } \\
\text { symptoms }\end{array}$ \\
\hline Theorell et al. 2011 & Age, gender, BMI, alcohol consumption, physical activity \\
\hline Gasa et al. 2011 & Age, gender, BMI, smoking habits \\
\hline Agrawal et al. 2012 & Age, gender, BMI, biceps skin fold thickness, PPNC, percent body fat, fat mass \\
\hline Hall et al. 2012 & $\begin{array}{c}\text { Gender, BMI, alcohol consumption, smoking habits, race, menopausal status, } \\
\text { education, marital status, health complaints, medications affect sleep, physical } \\
\text { activity }\end{array}$ \\
\hline
\end{tabular}

3 studies used standard PSG in detecting nocturnal IH, while 2 used pulse oximetry; 10 diagnosed MS according to NCEP ATPIII criteria, 2 according to Japanese criteria, and 1 according to International Diabetes Federation (IDF) guidelines. Table I lists detailed characteristics of the included studies. With regard to controlled confounding factors, 10 of 13 studies adjusted for BMI when calculating ORs; some studies also controlled for age, gender, smoking, alcohol consumption, physical activity, etc., as shown in Table II.

\section{Association between OSA and MS risk}

Obstructive sleep apnea was significantly associated with increased risk of MS (pooled OR = $1.72,95 \% \mathrm{Cl}: 1.31-2.66, p<0.001$; Figure 2), with evidence of between-study heterogeneity $\left(R^{2}=\right.$ $83.2 \%, p<0.001)$. The BMI-adjusted pooled OR was 1.97 (95\% Cl: $1.34-2.88, p<0.001)$.

To explore the source of heterogeneity, independent variables including mean age, sex ratio, mean BMI, geographic region ("Europe", "America", and "Asia"), OSA category ("binary", "continuous"), MS criteria (“NCEP ATPIII", "IDF”, and "Japanese criteria"), and study design ("case control", "cross sectional") were introduced into the univariate meta regression analysis. Among these, only the OSA category was significantly related to high heterogeneity $(p=0.018)$. Therefore, we presented the final results by subgroup analysis with reference to OSA category. The combined ORs between OSA and MS risk in binary and continuous categorized studies were 2.47 (95\% Cl: 1.61-3.77, $p<0.001)$ and 1.14 (95\% Cl: 0.98-1.34, $p=0.10$ ), respectively (Figure 3 ). The heterogeneity in the two subgroups decreased but could not be fully explained by the OSA category $(12=57 \%, p=0.03$ in the binary group and $R^{2}=63.3 \%, p=0.066$ in the continuous group).

Subgroup analyses were conducted to investigate the influence of study design, MS criteria, and method of diagnosis of OSA. Although these subgroup analyses were not particularly helpful in verifying the source of heterogeneity, the association between OSA and MS remained significant in all subgroups (Table III).

Publication bias was detected using Egger's test $(p<0.001)$, and the trim and fill method was used to add possible missing negative results. After four studies were included as possible missing studies, the publication bias disappeared (Figure 4), and the association between OSA and MS risk was still significant $(\mathrm{OR}=1.37,95 \% \mathrm{Cl}$ : 1.06-1.77, $p=0.016)$.

\section{Association between nocturnal IH and MS risk}

ODI and CT90, two nocturnal IH-measuring indexes that were available in the original studies, were also found to be associated with increased MS risk. The pooled OR for MS in individuals with 
Study ID

OR $(95 \% \mathrm{Cl}) \quad$ Weight $(\%)$

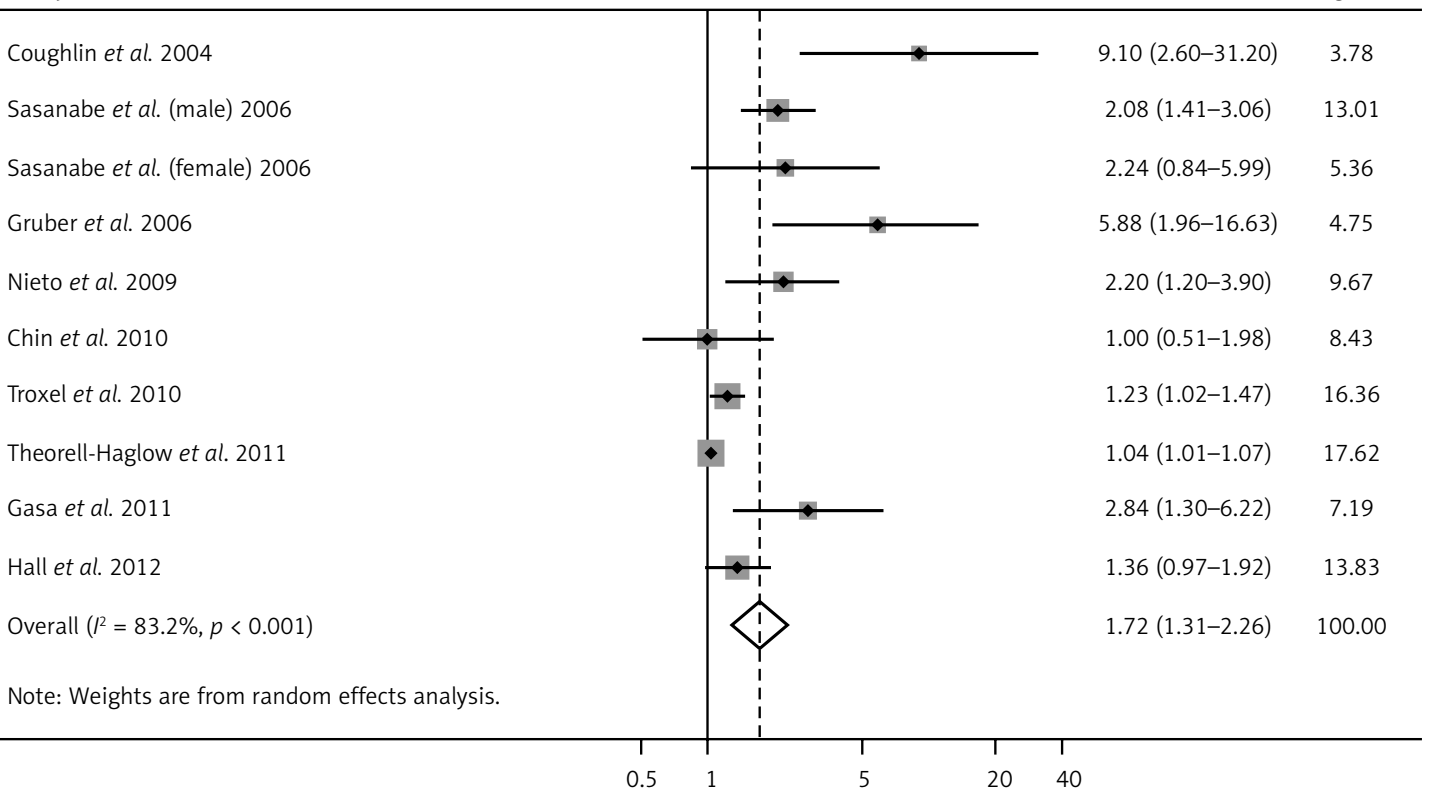

Figure 2. Forest plot summarizing the association between OSA and MS in the 10 included studies under the random effects meta-analysis. The sizes of the gray boxes in the figure are in proportion to the weight assigned to each study, whereas the point within each box is the OR reported by each study. The horizontal bar is the reported $95 \% \mathrm{Cl}$ for each OR

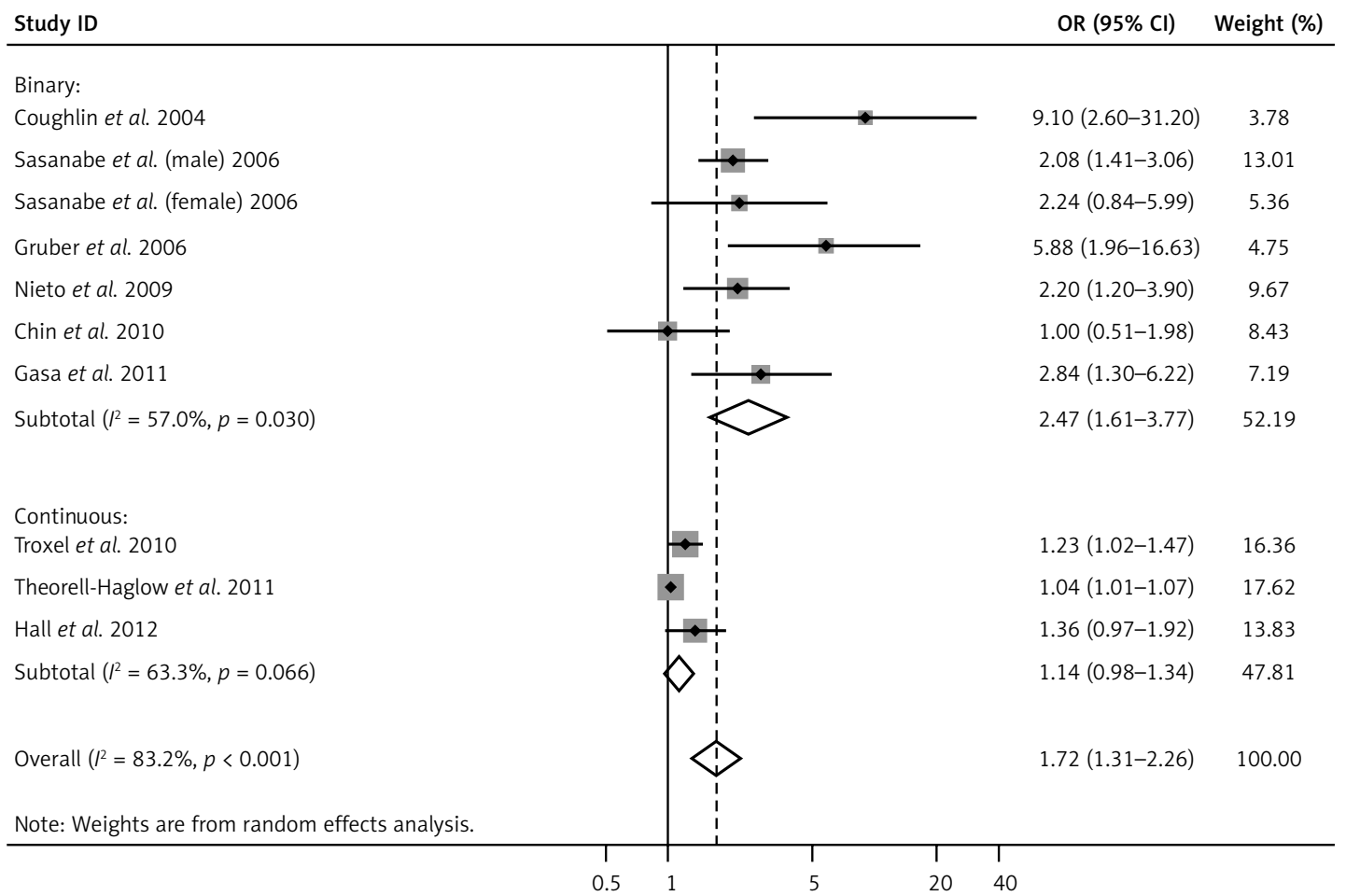

Figure 3. Forest plot summarizing the subgroup meta-analysis of the association between OSA and MS by OSA criteria (binary and continuous)

higher ODI was $1.96(95 \% \mathrm{Cl}: 1.30-2.96, p<0.001)$, with evidence of high heterogeneity $\left({ }^{2}=90.5 \%\right.$, $p<0.001$ ) (Figure $5 \mathrm{~A}$ ). The heterogeneity could not be explained by meta regression of any confounding factors including mean age, sex ratio, mean $\mathrm{BMI}$, geographic region, ODI category, MS criteria, study design, and method of diagnosis of ODI. The pooled OR for MS in individuals with higher CT90 was 1.05 (95\% Cl: 1.02-1.07, $p<0.001)$, with no evidence of variation $\left({ }^{2}=0 \%, p=0.612\right)$ (Figure $5 \mathrm{~B}$ ). Egger's test confirmed that there was no significant publication bias ( $p=0.392 / 0.967$ separately). When adjusted for BMI, both the associations between ODI and MS (pooled OR $=1.33,95 \% \mathrm{Cl}$ : 
Table III. Subgroup meta-analysis between OSA and MS

\begin{tabular}{|c|c|c|c|c|c|c|}
\hline Subgroup & No. of studies & OR & $95 \% \mathrm{Cl}$ & $P_{z}$ & $I^{2}$ & $P_{H}$ \\
\hline \multicolumn{7}{|l|}{ Study design: } \\
\hline Cross sectional & 8 & 2.18 & $1.35-3.53$ & 0.001 & $85 \%$ & $<0.001$ \\
\hline Case control & 2 & 1.26 & $1.07-1.48$ & 0.006 & 0 & 0.61 \\
\hline \multicolumn{7}{|l|}{ MS criteria: } \\
\hline NCEP ATPIII & 7 & 1.44 & $1.11-1.88$ & 0.006 & $79 \%$ & $<0.001$ \\
\hline IDF guidelines & 1 & 5.88 & $1.96-16.63$ & 0.002 & - & - \\
\hline Japanese criteria & 2 & 2.10 & $1.46-3.02$ & $<0.001$ & 0 & 0.89 \\
\hline \multicolumn{7}{|c|}{ Diagnostic method of OSA: } \\
\hline Standard PSG & 8 & 2.17 & $1.42-3.31$ & $<0.001$ & $86 \%$ & $<0.001$ \\
\hline Portable PSG & 2 & 1.21 & $1.01-1.45$ & 0.04 & 0 & 0.56 \\
\hline
\end{tabular}

OSA - obstructive sleep apnea, MS - metabolic syndrome, PSG - polysomnography, NCEP ATPIII - National Cholesterol Education Program Adult Treatment Panel III, IDF - International Diabetes Federation.

1.06-1.67, $p=0.01)$ and between CT90 and MS (pooled OR $=1.05,95 \% \mathrm{Cl}: 1.02-1.07, p<0.001$ ) remained significant.

\section{Quality assessment and sensitivity analysis}

Most of the studies revealed some potential risk of bias, as assessed by the Newcastle Ottawa Scale (Table IV). Regarding the selection of MS and controls, all the studies included MS samples that were non-representative of the general MS population, which could have led to potential selection bias. The definitions of MS in the 13 studies were all adequate. Controls all had no history of MS, but Sasanabe et al. [27], Gruber et al. [26], Gasa et al. [31], and Agrawal et al. [33] recruited hospital-based controls rather than population-based controls, who might have had lower comparability.

Regarding the assessment of OSA and nocturnal $\mathrm{IH}$, all studies but one [25] used the same method of detection of OSA in MS and control groups. However, unlike the 9 studies that applied standard PSG, 2 studies (Troxel et al. [30] and Chin et al. [29]) used portable PSG to separate OSA and non-OSA patients, and 2 studies, both from Muraki et al. [34], used pulse oximetry to detect nocturnal $\mathrm{IH}$. These kinds of methods could introduce a potential risk of misclassification bias.

Regarding the controlled variables in calculation of ORs, obesity was selected as the most important confounding factor. Overall, 10 studies controlled for obesity by adjusting for BMI, while Muraki et al. [34] and Throxel et al. [30] did not. Most of the studies controlled for some other factors (e.g., age, gender, smoking habits, alcohol consumption, physical activity) that might be related to MS risk (see Table II).

Sensitivity analysis by removal of any study from analyses of OSA and MS, ODI and MS,

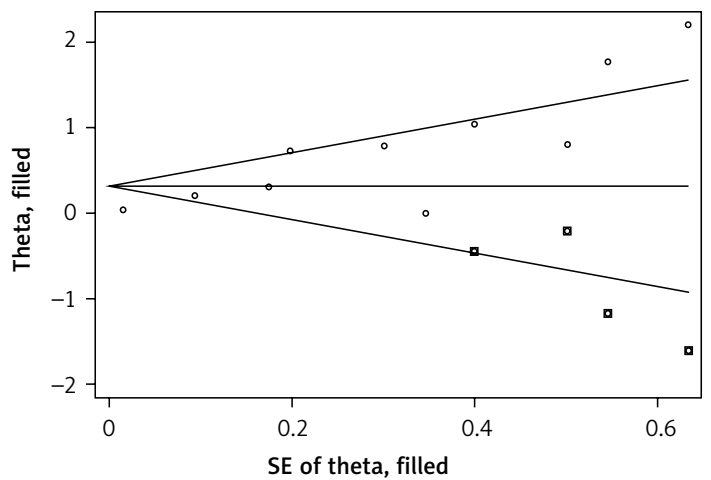

Figure 4. Funnel plot showed a publication bias but it could be corrected by trim and fill analysis

and CT90 and MS did not significantly subvert the present pooled analysis results (data not shown).

\section{Discussion}

\section{Summary of findings}

Overall, in the pooled analyses, subjects with OSA were at 1.72 times higher risk for MS. Nocturnal $\mathrm{IH}$, one of the most important pathogenic mechanisms in OSA, as estimated using ODI and CT90, was found to play a potential role in the association between OSA and risk of MS. Sensitivity analyses confirmed the stability of the conclusions. Furthermore, significant associations were also observed in analyses that considered the confounding effect of BMI.

Subgroup analysis that regarded a continuous variable (AHI) as the exposure factor did not reach significance, while the binary-categorized subgroup did. This may be due to the rather small dose cut-off for $\mathrm{AHI}$ in the former subgroup (e.g., per 1 unit [6, 32] or per 5 units [30]). 
A

Study ID

OR $(95 \% \mathrm{Cl}) \quad$ Weight $(\%)$

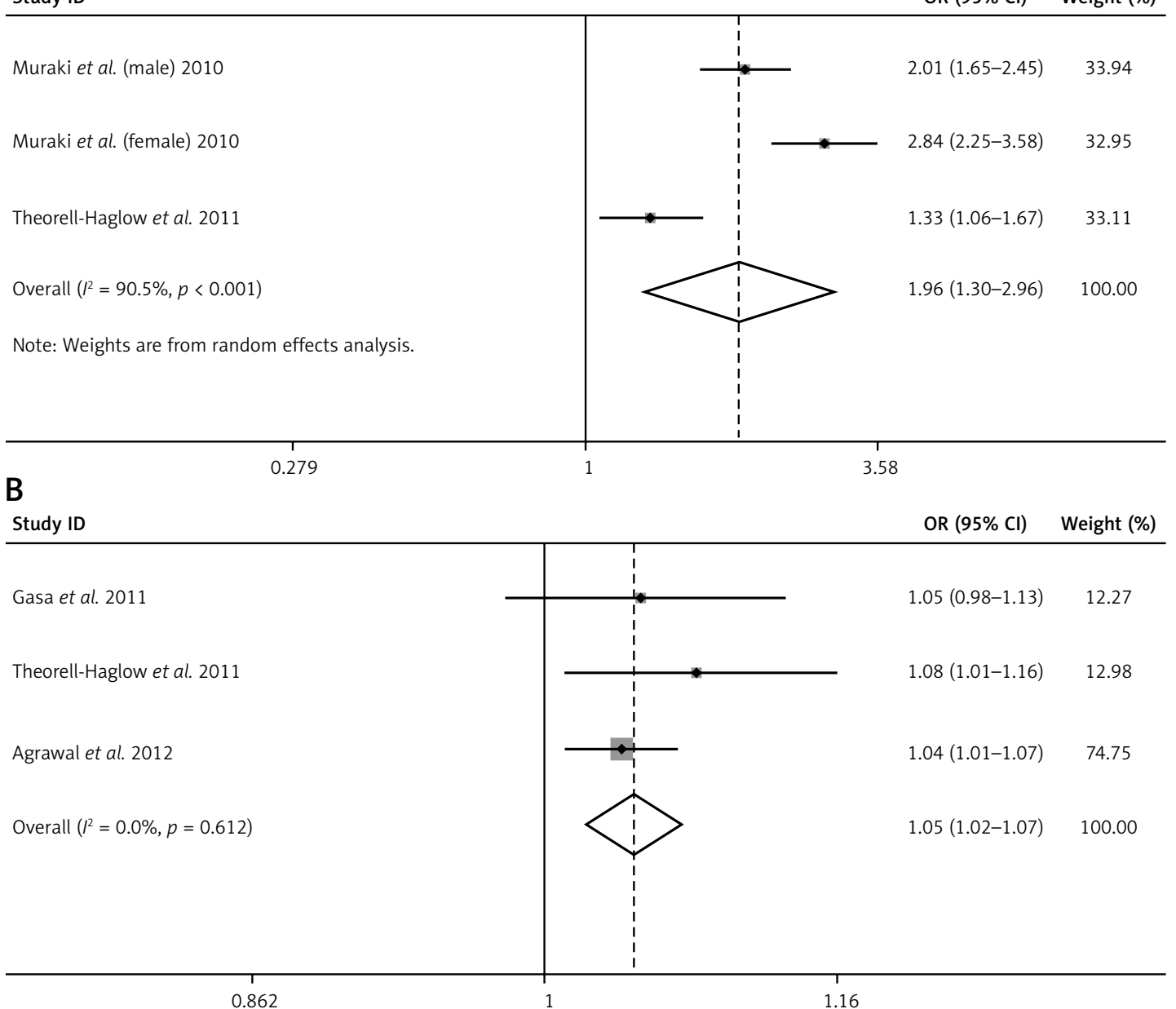

Figure 5. A - Forest plot summarizing the association between ODI and MS in the 3 included studies under the random effects meta-analysis. B - Forest plot summarizing the association between CT90 and MS in the 3 included studies under the fixed effects meta-analysis

Table IV. Study quality as assessed by the Newcastle Ottawa Scale

\begin{tabular}{|c|c|c|c|c|c|}
\hline Study & $\begin{array}{l}\text { Selection } \\
\text { (maximum, } \\
4 \text { asterisks) }\end{array}$ & $\begin{array}{c}\text { Comparability } \\
\text { (maximum, } \\
2 \text { asterisks) }\end{array}$ & $\begin{array}{l}\text { Exposure } \\
\text { (maximum, } \\
3 \text { asterisks) }\end{array}$ & Total score & Level \\
\hline Coughlin et al. 2004 & $* * *$ & ** & ** & 7 & 2 \\
\hline Sasanabe et al. (male) 2006 & ** & ** & $* * *$ & 7 & 2 \\
\hline Sasanabe et al. (female) 2006 & ** & ** & $* * *$ & 7 & 2 \\
\hline Gruber et al. 2006 & ** & ** & $* * *$ & 7 & 2 \\
\hline Nieto et al. 2009 & $* * *$ & $* \star$ & $* * *$ & 8 & 2 \\
\hline Chin et al. 2010 & $* *$ & $* *$ & $* *$ & 6 & 2 \\
\hline Muraki et al. (male) 2010 & $* * *$ & * & ** & 6 & 2 \\
\hline Muraki et al. (female) 2010 & $* * *$ & * & ** & 6 & 2 \\
\hline Troxel et al. 2010 & $* * *$ & * & $* *$ & 6 & 2 \\
\hline Theorell-Haglow et al. 2011 & $* * *$ & ** & $* * *$ & 8 & 2 \\
\hline Gasa et al. 2011 & ** & ** & $* * *$ & 7 & 2 \\
\hline Agrawal et al. 2012 & ** & ** & $* * *$ & 7 & 2 \\
\hline Hall et al. 2012 & $* * *$ & $\star *$ & $* * *$ & 8 & 2 \\
\hline
\end{tabular}




\section{Strengths and limitations}

To our knowledge, this is not only the first meta-analysis to quantitatively examine the evidence for an association between OSA and risk of MS, but also the first to clarify an association between nocturnal IH (ODI and CT90) and risk of MS, which suggested a potential mechanism for the association between OSA and risk of MS. Additionally, our meta-analysis systemically conducted an expansive literature search and finally included nearly 8,000 samples, pooled from 13 independent published studies. This greatly improved the statistical power of our findings, and the conclusions are more credible than those of individual studies. We also examined the influence of BMI on the associations between OSA, nocturnal $\mathrm{IH}$, and risk of MS.

Our findings appear to be supported by experimental studies mimicking the influence of OSA on MS risk. Nocturnal IH was found to be the most important potential mediating factor. Intermittent hypoxia can profoundly alter both acute and chronic glucose homeostasis. Sympathetic activation, decreased oxidative phosphorylation, leptin signaling, and growth hormone axis suppression are possible mediators [17, 42]. Regarding hyperlipidemia, $\mathrm{IH}$ increases the expression of sterol regulatory element binding protein 1 (SREBP-1), a key regulator of synthesis for several lipids, as well as a downstream enzyme of lipoprotein secretion, stearoyl-CoA desaturase 1 (SCD-1) [1315]. Weight gain under hypoxic conditions may be due to the expression of adipokines, cytokines, or hormones in adipose tissues, which may contribute systemically to the development of various features of metabolic syndrome and cardiovascular disease $[12,16]$. The IH causes elevated blood pressure during both sleep and wakefulness by mechanisms of sympathoexcitation, including augmentation of peripheral chemo reflex sensitivity and a direct effect on central sites of sympathetic regulation [18].

The results of this meta-analysis must be interpreted in the light of the following limitations. First, because of their observational nature, all of the included studies could not strictly control for confounding factors that may be inherent in the original studies, and the study design of cross-sectional and case-control studies made it impossible to address causality between OSA or nocturnal $\mathrm{IH}$ and MS as well as prospective cohort studies would. We conducted strict quality assessment of the selected studies according to the Newcastle Ottawa Scale, and all the included studies were of a high level of quality (level 2). We are confident that our pooled results have sufficient power to avoid a statistical type II error. Additionally, because obesity may be the most important confounding factor, we presented the BMI-adjusted pooled results. It was not possible to conduct a meta-analysis of prospective cohort studies or longitudinal studies regarding the treatment effect of OSA on MS due to a paucity of these studies. Therefore, future research should explore the possibility that a causal relationship may exist between OSA or nocturnal IH and MS.

Second, the included studies were performed in a limited number of countries, so the findings might not be applicable to populations of other ethnicities. Third, publication bias was found in the association between OSA and MS, which could possibly have affected the pooled results. However, we corrected for possible publication bias by modifying the results using the trim and fill method, and the association remained significantly positive.

Fourth, we found evidence of between-study heterogeneity in the association between OSA and MS and the association between ODI and MS: meta-regression and stratified analyses were only able to identify partial sources of variation in the former, and were unable to explain the variation in the latter. However, all of the results for ODI and MS were positive, and no publication bias was found. The remaining heterogeneity may be due to the complexity of other unknown confounding factors, especially selection bias.

Finally, relatively few studies have focused on the association between ODI, CT90, and MS. More high-quality studies involving a larger number of participants will be needed to further explore the association between nocturnal $\mathrm{IH}$ and MS as well as the causality between them.

In conclusion, our meta-analysis revealed that OSA might be an important risk factor for MS, independent of BMI. The significant association between nocturnal $\mathrm{IH}$ and risk of MS that was further found in the pooled analyses indicated that $\mathrm{IH}$ is a potential mechanism that could help to explain the influence of OSA on the development of MS. The findings of our study have great clinical significance: 1) doctors should screen and treat OSA in MS patients to help control MS, CVD, and all-cause mortality; and 2) consistently with previous findings, it is important to monitor and control all MS profiles after patients are diagnosed with OSA, to help reduce OSA-related cardio-metabolic complications and mortality. However, more largescale, well-designed prospective studies are still needed to confirm the causal relation between OSA, nocturnal IH, and MS.

\section{Acknowledgments}

Yingjun Qian and Huajun Xu are co-first authors. These authors contributed equally to this article. 


\section{Conflict interest}

The authors declare no conflict of interest.

\section{References}

1. Third Report of the National Cholesterol Education Program (NCEP) Expert Panel on Detection, Evaluation, and Treatment of High Blood Cholesterol in Adults (Adult Treatment Panel III) final report. Circulation 2002; 106: 3143-421.

2. Wu SH, Liu Z, Ho SC. Metabolic syndrome and all-cause mortality: a meta-analysis of prospective cohort studies. Eur J Epidemiol 2010; 25: 375-84.

3. Ertek S, Cicero A. Impact of physical activity on inflammation: effects on cardiovascular disease risk and other inflammatory conditions. Arch Med Sci 2012; 8: 794-804.

4. Wang F, Zhang L, Zhang Y, et al. Meta-analysis on night shift work and risk of metabolic syndrome. Obes Rev 2014; 15: 709-20.

5. Xi B, He D, Zhang M, Xue J, Zhou D. Short sleep duration predicts risk of metabolic syndrome: a systematic review and meta-analysis. Sleep Med Rev 2014; 18: 293-7.

6. Hall MH, Okun ML, Sowers M, et al. Sleep is associated with the metabolic syndrome in a multi-ethnic cohort of midlife women: the SWAN Sleep Study. Sleep 2012; 35: 783-90.

7. Remmers JE, deGroot WJ, Sauerland EK, Anch AM. Pathogenesis of upper airway occlusion during sleep. J Appl Physiol Respir Environ Exerc Physiol 1978; 44: 931-8.

8. Young T, Finn L, Peppard PE, et al. Sleep disordered breathing and mortality: eighteen-year follow-up of the Wisconsin sleep cohort. Sleep 2008; 31: 1071-8.

9. Kostapanos MS, Mikhailidis DP, Elisaf MS, Steiropoulos P, Papanas N. Obstructive sleep apnoea syndrome and cardiovascular risk. Arch Med Sci 2012; 8: 1115-6.

10. Wilcox I, McNamara SG, Collins FL, Grunstein RR, Sullivan CE. "Syndrome Z": the interaction of sleep apnoea, vascular risk factors and heart disease. Thorax 1998; 53 Suppl. 3: S25-8.

11. Lavie L. Oxidative stress inflammation and endothelial dysfunction in obstructive sleep apnea. Front Biosci (Elite Ed) 2012; 4: 1391-403.

12. Alam I, Lewis K, Stephens JW, Baxter JN. Obesity, metabolic syndrome and sleep apnoea: all pro-inflammatory states. Obes Rev 2007; 8: 119-27.

13. Li J, Grigoryev DN, Ye SQ, et al. Chronic intermittent hypoxia upregulates genes of lipid biosynthesis in obese mice. J Appl Physiol (1985) 2005; 99: 1643-8.

14. Li J, Savransky V, Nanayakkara A, Smith PL, O'Donnell CP, Polotsky VY. Hyperlipidemia and lipid peroxidation are dependent on the severity of chronic intermittent hypoxia. J Appl Physiol (1985) 2007; 102: 557-63.

15. Li J, Thorne LN, Punjabi NM, et al. Intermittent hypoxia induces hyperlipidemia in lean mice. Circ Res 2005; 97: 698-706.

16. Phillips BG, Kato M, Narkiewicz K, Choe I, Somers VK. Increases in leptin levels, sympathetic drive, and weight gain in obstructive sleep apnea. Am J Physiol Heart Circ Physiol 2000; 279: H234-7.

17. Polotsky VY, Li J, Punjabi NM, et al. Intermittent hypoxia increases insulin resistance in genetically obese mice. J Physiol 2003; 552: 253-64.

18. Sunderram J, Androulakis IP. Molecular mechanisms of chronic intermittent hypoxia and hypertension. Crit Rev Biomed Eng 2012; 40: 265-78.
19. Peppard PE, Young T, Palta M, Skatrud J. Prospective study of the association between sleep-disordered breathing and hypertension. N Engl J Med 2000; 342: 1378-84.

20. Varounis C, Katsi V, Kallikazaros IE, et al. Effect of CPAP on blood pressure in patients with obstructive sleep apnea and resistant hypertension: a systematic review and meta-analysis. Int J Cardiol 2014; 175: 195-8.

21. Tan KC, Chow WS, Lam JC, et al. HDL dysfunction in obstructive sleep apnea. Atherosclerosis 2006; 184: 377-82.

22. Lin MT, Lin HH, Lee PL, et al. Beneficial effect of continuous positive airway pressure on lipid profiles in obstructive sleep apnea: a meta-analysis. Sleep Breath 2015; 19: 809-17.

23. Al-Delaimy WK, Manson JE, Willett WC, Stampfer MJ, $\mathrm{Hu}$ FB. Snoring as a risk factor for type II diabetes mellitus: a prospective study. Am J Epidemiol 2002; 155: 387-93.

24. Chen L, Pei JH, Chen HM. Effects of continuous positive airway pressure treatment on glycaemic control and insulin sensitivity in patients with obstructive sleep apnoea and type 2 diabetes: a meta-analysis. Arch Med Sci 2014; 10: 637-42.

25. Coughlin SR, Mawdsley L, Mugarza JA, Calverley PM, Wilding JP. Obstructive sleep apnoea is independently associated with an increased prevalence of metabolic syndrome. Eur Heart J 2004; 25: 735-41.

26. Gruber A, Horwood F, Sithole J, Ali NJ, Idris I. Obstructive sleep apnoea is independently associated with the metabolic syndrome but not insulin resistance state. Cardiovasc Diabetol 2006; 5: 22.

27. Sasanabe R, Banno K, Otake K, et al. Metabolic syndrome in Japanese patients with obstructive sleep apnea syndrome. Hypertens Res 2006; 29: 315-22.

28. Nieto FJ, Peppard PE, Young TB. Sleep disordered breathing and metabolic syndrome. WMJ 2009; 108: 263-5.

29. Chin K, Oga T, Takahashi K, et al. Associations between obstructive sleep apnea, metabolic syndrome, and sleep duration, as measured with an actigraph, in an urban male working population in Japan. Sleep 2010; 33: 89-95.

30. Troxel WM, Buysse DJ, Matthews KA, et al. Sleep symptoms predict the development of the metabolic syndrome. Sleep 2010; 33: 1633-40.

31. Gasa M, Salord N, Fortuna AM, et al. Obstructive sleep apnoea and metabolic impairment in severe obesity. Eur Respir J 2011; 38: 1089-97.

32. Theorell-Haglow J, Berne C, Janson C, Lindberg E. The role of obstructive sleep apnea in metabolic syndrome: a population-based study in women. Sleep Med 2011; 12: 329-34.

33. Agrawal S, Sharma SK, Sreenivas V, Lakshmy R, Mishra HK. Stepped approach for prediction of syndrome $Z$ in patients attending sleep clinic: a north Indian hospital-based study. Sleep Breath 2012; 16: 621-7.

34. Muraki I, Tanigawa T, Yamagishi K, et al. Nocturnal intermittent hypoxia and metabolic syndrome; the effect of being overweight: the CIRCS study. J Atheroscler Thromb 2010; 17: 369-77.

35. Moher D, Liberati A, Tetzlaff J, Altman DG. Preferred reporting items for systematic reviews and meta-analyses: the PRISMA statement. Int J Surg 2010; 8: 336-41.

36. Wells GA SB, O'Connell D, et al. The Newcastle-Ottawa Scale (NOS) for assessing the quality of nonrandomised studies in meta-analyses. Ottawa, Canada. Available at: http://www.ohri.ca/programs/clinical_epidemiology/ oxford.htm.

37. Hamling J, Lee P, Weitkunat R, Ambuhl M. Facilitating meta-analyses by deriving relative effect and precision 
estimates for alternative comparisons from a set of estimates presented by exposure level or disease category. Stat Med 2008; 27: 954-70.

38. Higgins JP, Thompson SG. Quantifying heterogeneity in a meta-analysis. Stat Med 2002; 21: 1539-58.

39. Rosenberg MS. The file-drawer problem revisited: a general weighted method for calculating fail-safe numbers in meta-analysis. Evolution 2005; 59: 464-8.

40. Egger M, Smith GD. Bias in location and selection of studies. BMJ 1998; 316: 61-6.

41. Duval S, Tweedie R. Trim and fill: a simple funnel-plotbased method of testing and adjusting for publication bias in meta-analysis. Biometrics 2000; 56: 455-63.

42. liyori N, Alonso LC, Li J, et al. Intermittent hypoxia causes insulin resistance in lean mice independent of autonomic activity. Am J Respir Crit Care Med 2007; 175: 851-7. 